Research Paper

\title{
The habenula as a critical node in chronic stress-related anxiety
}

\author{
Luis R. Jacinto, Rui Mata, Ashley Novais, Fernanda Marques, Nuno Sousa * \\ Life and Health Sciences Research Institute, School of Medicine, University of Minho, Braga, Portugal \\ ICVS/3B's - PT Government Associate Laboratory, Braga/Guimarães, Portugal
}

\section{A R T I C L E I N F O}

Article history:

Received 13 August 2016

Received in revised form 2 December 2016

Accepted 5 December 2016

Available online 7 December 2016

\section{Keywords:}

Habenula

Stress

Anxiety

Stereology

Corticosterone

\begin{abstract}
A B S T R A C T
The habenula is activated in response to stressful and aversive events, resulting in exploratory inhibition. Although possible mechanisms for habenula activation have been proposed, the effects of chronic stress on the habenular structure have never been studied. Herein, we assessed changes in volume, cell density and dendritic structure of habenular cells after chronic stress exposure using stereological and 3D morphological analysis. This study shows for the first time that there is a hemispherical asymmetry in the medial habenula (MHb) of the adult rat, with the right $\mathrm{MHb}$ containing more neurons than its left counterpart. Additionally, it shows that chronic stress induces a bilateral atrophy of both the MHb and the lateral habenula (LHb). This atrophy was accompanied by a reduction of the number of neurons in the right $\mathrm{MHb}$ and the number of glial cells in the bilateral LHb, but not by changes in the dendritic arbors of multipolar neurons. Importantly, these structural changes were correlated with elevated levels of serum corticosterone and increased anxious-like behavior in stressed animals. To further assess the role of the habenula in stress-related anxiety, bilateral lesions of the LHb were performed; interestingly, in lesioned animals the chronic stress protocol did not trigger increases in circulating corticosterone or anxious-like behavior. This study highlights the role of the habenula in the stress responses and how its subregions are structurally impacted by chronic stress with physiological and behavioral consequences.
\end{abstract}

(c) 2016 Published by Elsevier Inc.

\section{Introduction}

The habenula is a small bilateral brain region that together with the pineal gland forms the epithalamus and is phylogenetically preserved in almost all vertebrates (Andres et al., 1999; Stephenson-Jones et al., 2012). The habenular nuclei, which consist of the medial and lateral sub-regions ( $\mathrm{MHb}$ and $\mathrm{LHb}$, respectively), have been described as a relay interface between the basal ganglia and the limbic system since forebrain projections from both converge there (Herkenham and Nauta, 1977) and are able to modulate downstream limbic midbrain dopamine and serotonin circuits (Hikosaka et al., 2008; Hikosaka 2010; Stephenson-Jones et al., 2012; Zhao et al., 2015). This modulation can both promote or inhibit release of dopamine and serotonin in the brain since the habenula is strongly connected with the ventral tegmental area (VTA) - via the rostromedial tegmental nucleus (rMTG) - and the raphe nucleus (RN) - via the interpeduncular nucleus (IPN) (Christoph et al., 1986; Ferraro et al., 1996; Lecourtier et al., 2008; Zhao et al., 2015) -, the major sources of dopaminergic and serotonergic projections in the brain, respectively (Vertes, 1991; Russo and Nestler, 2013). Although the LHb appears to be the only habenula sub-region capable of directly controlling dopamine release, serotonergic modulation

\footnotetext{
* Corresponding author at: Life and Health Sciences Research Institute (ICVS), School of Medicine, Universidade do Minho, Campus de Gualtar, 4710-103 Braga, Portugal.

E-mail address: njcsousa@med.uminho.pt (N. Sousa).
}

is likely more complex as both structures exert influence on the IPN the MHb directly and the LHb indirectly - and the RN (Hikosaka et al., 2008; Hikosaka, 2010; Stephenson-Jones et al., 2012; Zhao et al., 2015), suggesting that the combined output of both structures is particularly critical for the serotonergic system. Moreover, the MHb also projects to the LHb (Kim and Chang, 2005) and both sub-structures share several efferent inputs (Herkenham and Nauta, 1977) further highlighting the complexity of the combined net output of the habenula This critical modulatory role of the monoamines circuits has been linked to several different behaviors and conditions (Hikosaka, 2010; Fakhoury and López, 2014; Proulx et al., 2014), therefore attracting increased attention from researchers focusing on mood disorders, depression and stress (Hikosaka, 2010).

The particular involvement of the habenula in the stress response was initially attributed to the observation that habenular neurons were activated by a variety of stressors (Wirtshafterm et al., 1994) and aversive/unpleasant stimuli including both the presence of punishment and the absence of reward (Matsumoto and Hikosak, 2009). Additionally, stimulation of the habenula produced autonomic responses similar to those observed in emotional stress (Ootsuka and Mohammed, 2015) and LHb activity was correlated with behavioral inhibition in normal and stressful contexts (Lee and Huang, 1988). This inhibitory role has been linked to the modulation of dopamine and serotonin release in the brain and has been additionally shown in several lesion (Murphy et al., 1996; Yang et al., 2008; Tian and Uchida, 2015), 
excitation (Christoph et al., 1986; Ji and Shepard, 2007) and inhibition (Winter et al., 2011; Sachs et al., 2015) and optogenetic (Stamatakis and Stuber, 2012; Amo et al., 2014) studies.

Chronic exposure to stress leads to monoamines dysfunction with behavioral consequences (Chaouloff et al., 1999; Pani et al., 2000; Bambico et al., 2009) that mimic those described for overactivation of the habenula. Moreover, several depression studies that use chronic stress exposure as a model have shown an involvement of the habenula in the dysregulation of the monoamines circuits with functional and behavioral consequences (Christensen et al., 2013; Aizawa et al., 2013; Proulx et al., 2014). More strikingly, it was also shown that habenula-induced monoamine dysfunction could be one of the causes for increased susceptibility to stress (Sachs et al., 2015). Yet, and although some mechanisms for habenula overactivation have been proposed (Aizawa et al., 2013; Li et al., 2013; Cui et al., 2014), the consequences of chronic exposure to stress on habenular structure have never been explored.

Here, we assessed how chronic exposure to stress could lead to changes in volume, cell density and dendritic structure of habenular cells by stereological and 3D morphological analysis of Giemsa and Golgi stained LHb and MHb sections. We not only present, for the first time, an unbiased estimation of the total number of neuronal cells in the MHb and LHb and glial cells in the LHb of the adult rat in both brain hemispheres, but also how chronic stress can disrupt the basal structure. Anxiety-related disorders - despite of their clinical importance - have received far less attention than depression in previous habenula studies; therefore, we additionally assessed if habenular integrity was necessary for the physiological and behavioral expression of stress-related anxiety by exposing habenula-lesioned animals to a chronic stress protocol and then to an anxiogenic environment. Considering it had been shown that lesions of the LHb after a stress protocol could alleviate some of the immediate behavioral and physiological symptoms of depressive-like behavior (Yang et al., 2008) we also hypothesized that ablation of the LHb previous to the stress exposure could block the escalation of stress-related anxiety symptoms due to stress exposure.

\section{Materials and methods}

\subsection{Animals}

A total of 36 Male Wistar-Han rats (Charles River laboratories, Barcelona, Spain), weighting 300-400 g and aged 12-16 weeks were used in this study. Animals were pair-housed under the following laboratory conditions: room temperature of $22{ }^{\circ} \mathrm{C}$, relative humidity of $55 \%, 12 \mathrm{~h}$ light cycle beginning at $8 \mathrm{AM}$ and food and water ad libitum. All procedures performed in studies involving animals were in accordance with the ethical standards of the institution or practice at which the studies were conducted. Experiments were conducted in accordance with European Union Directive 2010/63/EU and the Portuguese regulations and laws on the protection of animals used for scientific purposes of the Ministry for Agriculture, Rural Development and Fishing. This study was approved by the Portuguese Veterinary General Direction, Direção Geral de Alimentação e Veterinária (DGAV).

Animals were divided into 4 groups: control $(\mathrm{n}=12)$, stress $(\mathrm{n}=$ $12)$, lesion-control $(n=6)$ and lesion-stress $(n=6)$.

\subsection{Surgery/electrolytic lesion}

Animals from the lesion (lesion-control and lesion-stress) groups were subjected to bilateral electrolytic ablation of the LHb by passing a current through a concentric tungsten electrode (PI2CEA10, Microprobes, Gaithersburg, MD, USA). An adaptation of the lesion protocol of (Haack et al., 2014) was used: since the habenula has a quasi-tubular form, anterior and posterior lesions were performed in order to increase the extent of the lesion in the targeted area and reduce the damage to neighboring regions. Coordinates for the sites were: -3.0 and
$-3.7 \mathrm{~mm} \mathrm{AP}, \pm 0.8 \mathrm{~mm} \mathrm{ML}$, and $-5.4 \mathrm{~mm} \mathrm{DV}$, from bregma. Lesions were produce by passing a current of $1 \mathrm{~mA}$ for 6 and $10 \mathrm{~s}$ in the most anterior and most posterior sites, respectively. Lesions were confirmed in Cresyl-violet stained $30 \mu \mathrm{m}$ slices from the post-mortem brains. Supplementary Fig. 1 shows a schematic representation of the average lesion extent on all lesioned animals. Although the electrolytic lesions were largely confined to the $\mathrm{LHb}$, in some sites the lesion extended beyond the LHb to surrounding structures, including the MHb. Three rats (2 from the lesion-control group and 1 from the lesion-stress group) were removed from further data analysis due to poorly targeted lesions.

Animals were allowed to recover from surgery for 1-2 weeks.

\subsection{Stress protocol}

Rats from the stress groups (stress, and lesion-stress) were then exposed to a chronic unpredictable stress (CUS) protocol, described elsewhere (Cerqueira et al., 2007), for 28 days. Importantly, exposure to this CUS protocol is known to induce anxiety-like behavior (Pêgo et al., 2008; Jacinto et al., 2013). Briefly, stressed animals were exposed to a daily stressor (up to $1 \mathrm{~h}$ a day). In order to avoid adaptation the stressor applied was different every day and presented at a different and random hour of the day. Five different stressors were used: restraint, noise, shaking, cold water and hot air stream. All stressors were applied in a separate experimental room from where the animals of both groups were housed. Control group animals (control and lesioncontrol) were handled for the same time during the same period.

On the day following the end of the stress protocol blood samples were drawn from all animals (from the 4 groups) via tail venipuncture for serum corticosterone levels assessment. Blood samples were collected in the morning ( $8 \mathrm{AM}$ ). The samples were centrifuged at $13000 \mathrm{rpm}$ for $10 \mathrm{~min}$. Serum was extracted and stored at $-80{ }^{\circ} \mathrm{C}$ for posterior analysis. Serum corticosterone levels were measured by immunoassay using a commercially available ELISA kit (Corticosterone ELISA kit, Enzo Life Sciences, Farmingdale, NY, USA).

\subsection{Behavior}

Following one day of rest after blood collection, animals from all groups were exposed to the Elevated-Plus Maze (EPM) test for 5 min. The EPM is a validated test to assess anxiety-like behavior in rodents and the protocol has been described elsewhere (Sousa et al., 2006). Briefly, the test apparatus consists of an elevated plus shape where two of the plus' arms are surrounded by high walls and the two other are not. The test explores the conflict of appetitive and aversive motivations to explore novel environments and avoid brightly lit and elevated spaces, respectively, Responsiveness to the anxiety-inducing environment was measured as the time animals spent exploring the open arms of the EPM, regarded as most aversive areas of the test. Animals which present higher anxiety-levels (or higher response to anxiety-inducing stimuli) tend to spend less time exploring the open arms (Pêgo et al., 2008). Since the EPM was performed at different times across different groups and subtle environmental variations may impact on behavior - even though the order of the animals was randomized -, the time spent in the open arms was transformed in a z-score when all groups were compared. The averaged non-z-transformed data is also shown as Supplementary Fig. 2.

\subsection{Stereology}

Stereological procedures were similar to the ones described in (Cerqueira et al., 2007). On the day following the behavioral testing part of the rats from the control $(n=6)$ and stress $(n=6)$ groups were anaesthetized with an overdose of pentobarbital injected intraperitoneally and perfused transcardially with a fresh solution of $0.9 \%$ saline followed by a fixative solution (4\% paraformaldehyde). The brains were removed, cut in a $5 \mathrm{~mm}$ section that included all of the habenula, with 
the help of a brain slicer (Zivic, Pittsburgh, PA, USA) and placed in fixative solution for at least 3 weeks. The blocks were then sequentially dehydrated in alcohol solutions and then embedded in glycolmetha crylate (Tecnovit 7100, Heraeus Kulzer, Werheim, Germany). The glycolmethacrylate-embedded blocks were marked for laterality with a cut on the top-right side of the block with a blade and finally cut in a microtome in $30 \mu \mathrm{m}$ coronal sections. Every section was collected on noncoated glass slides, stained with Giemsa (Sigma-Aldrich, SaintLouise, MO, USA) and mounted with Entellan (Merck, Darmstad, Germany).

\subsubsection{Region boundaries}

$\mathrm{MHb}$ and $\mathrm{LHb}$ regions were demarked based on the rat brain atlas of Paxinos and Watson (2006) and previous stereological and patch clamp works (Kim and Chang, 2005; Zhang and Oorschot, 2006). The MHb consisted of a small region with closely packed cells surrounded by endothelial cells bordering the third ventricle and neighbored by the $\mathrm{LHb}$ to the side opposite the ventricle. The LHb was larger than the MHb in the majority of sections although presenting cells that were more sparsely distributed. The differential packaging of cells stained the $\mathrm{MHb}$ much more darkly than the LHb. Both MHb and LHb were bordered on top by the stria medularis on the majority of sections and were clearly separated from the dorsal thalamus at the bottom by a thin layer of cells, likely belonging to the fasciculus retroflexus. Supplementary Fig. 3 shows demarked $\mathrm{MHb}$ and $\mathrm{LHb}$ regions on exemplificative sections.

\subsubsection{Cell identification}

In both the MHb and the LHb it was possible to clearly identify two distinct cell populations: glial cells and neurons (Zhang and Oorschot, 2006). The distinct populations were divided based on morphological characteristics such as size, distribution of euchromatin, visibility of nucleolus and nucleoplasm. Glial cells displayed round, smaller and darkly stained nucleus (due to the presence of heterochromatin aggregates) without a visible nucleolus or nucleoplasm (Davanlou and Smith 2004; Zhang and Oorschot, 2006; García-Amado and Prensa, 2012) - although microglia may present an elongated form with dense peripheral chromatin (Davanlou and Smith 2004; Pelvig et al., 2008). Neurons, on the other hand, presented larger, spherical or ovoidal, nucleus with a pale and homogeneous nucleoplasma almost always with a clear nucleolus nucleoplasm (Davanlou and Smith 2004; Zhang and Oorschot, 2006; García-Amado and Prensa, 2012). The distinction between different types of neurons or glial cells was not taken into account and would only be accurate with additional immunohistochemistry staining, which was not performed - however, in an initial pilot stereological study in the LHb no differences were seen on neuronal cell numbers between control and stressed rats and an additional stereological pilot with GFAP stained LHb sections showed a reduction on the number of stained cells in stressed rats (data not shown). Endothelial cells were also observed, especially in the MHb lining the border with the ventricle, presenting a curved or triangular shape and without visible chromatin or nucleolus (Davanlou and Smith, 2004), but were not counted. Cells which raised doubts regarding their type were counted separately but accounted for $<1 \%$ of the total number of cells counted on each rat. Supplementary Fig. 4 shows different cell types identified through morphology on the LHb.

\subsubsection{Stereological procedures}

Volume and cell number estimations were performed using Stereo Investigator software (MBF Bioscience, Williston, VT, USA) and a camera (DXC-390; Sony, Tokyo, Japan) attached to a motorized microscope (Axioplan 2; Carl Zeiss, Oberkochen, Germany). Volume estimation was based on planimetric data from the demarked regions of each section. Although this estimate is not unbiased it can provide a good estimation of volume given that the region of interest is properly delimitated (Cotter et al., 1999; Acer et al., 2008) and has been used successfully in rodent studies (Healy-Stoffel et al., 2001). Average cell numbers for each brain and region were estimated using the optical fractionator method, described elsewhere (West et al., 1991). The optical fractionator method allows the unbiased estimation of the total number of cells from thick sections, sampled in a predetermined sequential manner. Each selected section was randomly sampled by a set of unbiased virtual 3-D counting boxes covering each region of interest with a uniform distanced between counting sites in $\mathrm{X}, \mathrm{Y}$ directions where cells were counted through a thickness of $20 \mu \mathrm{m}$. For both the $\mathrm{MHb}$ and the LHb each fourth section was used. A counting frame of $20 \times 20 \mu \mathrm{m}$ and $30 \times 30 \mu \mathrm{m}$ was used for the MHb and LHb respectively. The X, Y spacing of each counting frame was $90 \times 90 \mu \mathrm{m}$ and $110 \times 110$ $\mu \mathrm{m}$ for the MHb and LHb respectively. Cells were counted within each counting frame according to pre-defined counting rules (West et al., 1991). Neurons and glial cells that fell within the description above were labeled accordingly.

Coefficients of error for the cell number estimates were automatically calculated according to the formulas of HJG et al. (1999) and were within the optimal range $(<0.10)$ for all rats, except for the estimation glial cell numbers in $\mathrm{MHb}$ in one-third of the rats and thus were not taken into consideration. Estimated coefficients of error for the planimetric volume estimates were also under 0.10 .

\subsection{Morphological analysis of dendritic arborization}

\subsubsection{Golgi processing}

One day after behavior, additional control $(n=6)$ and stressed rats $(n=6)$ were transcardialy perfused with $0.9 \%$ saline under pentobarbital anesthesia and brains were removed for Golgi-Cox staining (Gibb and Kolb, 1986). Brains were immersed in Golgi-Cox solution (a 1:1 solution of $5 \%$ potassium dichromate; Merck) and $5 \%$ mercuric chloride (Merck) diluted 4:10 with 5\% potassium chromate (Merck) for 14 days; then transferred to $30 \%$ sucrose solution where they were kept in the dark and at $4{ }^{\circ} \mathrm{C}$ until processing. Coronal vibratome $200 \mu \mathrm{m}$ thick sections were collected and blotted dry onto clean slides, alkalinized in 18.7\% ammonia, developed in Dektol (Kodak, Linda-aVelha, Portugal), fixed, dehydrated through a graded series of ethanol, cleared in xylene, mounted with Entellan (Merck) and coverslipped.

\subsubsection{Morphometrical analysis}

Multipolar habenular neurons were chosen randomly in the LHb within the regional boundaries previously described in the stereology section brain. 3-4 neurons were counted per brain. Note that the low number of neurons counted per brain is due to the fact that Golgi staining of habenular neurons is technically challenging as few neurons are stained through this technique, even using modified protocols as described below. The criteria to choose Golgi impregnated neurons were the same as described previously (Uylings et al., 1986): dendritic branches that were not incomplete, broken or non-impregnated; dendrites that did not show overlap with other branches; neurons that were visually well isolated. Eighteen to 20 neurons per experimental group were studied. For each selected neuron, all branches of the dendritic tree were reconstructed at $600 \times$ magnification using a motorized microscope (BX51; Olympus, Tokyo, Japan) with oil objectives attached to a camera (MBF Bioscience) and using the Neurolucida software (MBF Bioscience). Neuron morphology in the habenula is diverse (Kim and Chang, 2005) and for the sake of consistency only multipolar neurons with 3 to 6 dendrites were drawn. The total dendritic length of each neuron was calculated since it can be used as a measure of neuron plasticity, reflecting increased probability of inter-neuronal connection (Sala and Segal, 2014).

\subsection{Statistics}

Multi-group comparisons (z-time in EPM open arms and corticosterone for all animals) were performed with one-way analysis of variance 
(ANOVA) followed by post-hoc pairwise comparisons using Tukey's HSD test. Two-way ANOVA was used to assess interactions between control/ stress and left/right for stereology estimates followed by post-hoc pairwise comparisons using Tukey's HSD test. Comparisons of control/ stress for the morphology data were done by Welch's $t$-test. All datasets were tested for normality using the Shapiro-Wilk test. Results are expressed as mean \pm standard error of the mean (sem).

Goodness of fit and significance of correlations between corticosterone and volume and cell number estimates were obtained by linear regression.

\section{Results}

\subsection{Chronic stress impacts on the structure of the MHb and $L H b$}

A group of rats $(n=6)$ was exposed to a stress protocol for 28 days while a group of control animals $(n=6)$ was handled during the same period. At the end of the 28 days blood was drawn for corticosterone assessment and two days after animals were exposed to the EPM, an anxious-behavior inducing arena (Pêgo et al., 2008). See Methods section for details.
Following exposure to the EPM, the brains of all animals were processed for stereological assessment of volumes and neuronal and glial cell numbers of the bilateral LHb and MHb. Control rats displayed an $\mathrm{MHb}$ of equal volume on both hemispheres, but with significantly more neurons in the right $\mathrm{MHb}$ (interaction $\mathrm{F}(1,20)=7.62 ; \mathrm{p}<0.01$; post-hoc pairwise comparison $\mathrm{p}=0.04$; Fig. $1 \mathrm{a}$ ). Chronically stressed rats displayed a significant reduction in the volumes of the bilateral $\mathrm{MHb}$ and LHb when compared with controls (MHb: $\mathrm{F}(1,20)=50.85$, $\mathrm{p}<0.0001$; LHb: $\mathrm{F}(1,20)=26.62, \mathrm{p}<0.0001$; post-hoc comparison $\mathrm{p}<0.01$ for all areas; Fig. 1a).

The observed volumetric reduction was paralleled by a significant reduction of the total number of cells in the right $\mathrm{MHb}$ and the $\mathrm{LHb}$ in both hemispheres (MHb: $\mathrm{F}(1,20)=17,45, \mathrm{p}=0.0005$; post-hoc comparison (MHb right) $\mathrm{p}<0.001$; LHb: $\mathrm{F}(1,20)=119.6, \mathrm{p}<0.0001$; post-hoc comparison (LHb left and right) $\mathrm{p}<0.0001$; Fig. $1 \mathrm{~b}$ ). The reduction in the total number of cells in the right $\mathrm{MHb}$ was due to a significant reduction of neurons $(\mathrm{F}(1,20=13.05, \mathrm{p}<0.01$; post-hoc comparison $\mathrm{p}<0.01$; Fig. 1c), while the reduction observed in the $\mathrm{LHb}$ was due to a significant reduction of glial cells $(F(120)=32.07$, $\mathrm{p}<0.0001$; post-hoc comparison, for both left and right, $\mathrm{p}<0.01$; Fig. $1 \mathrm{~d})$. Moreover, the volumetric changes could not be attributed to
A

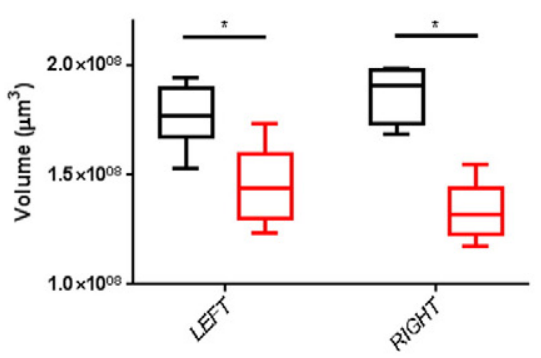

B

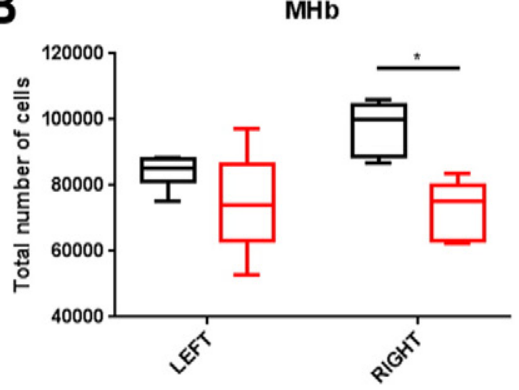

c

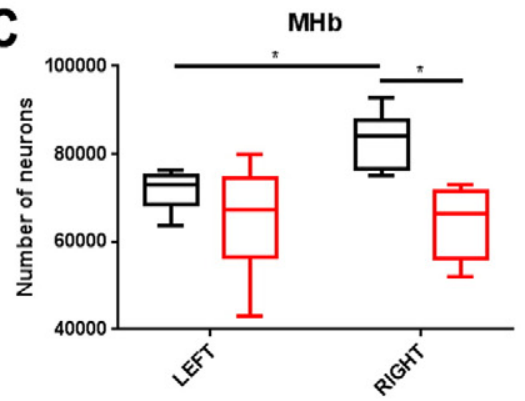

LHb

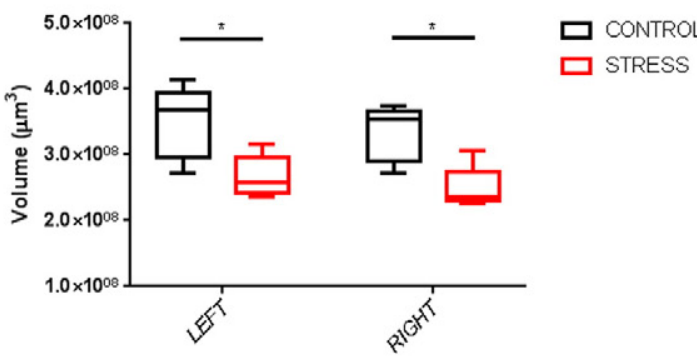

LHb

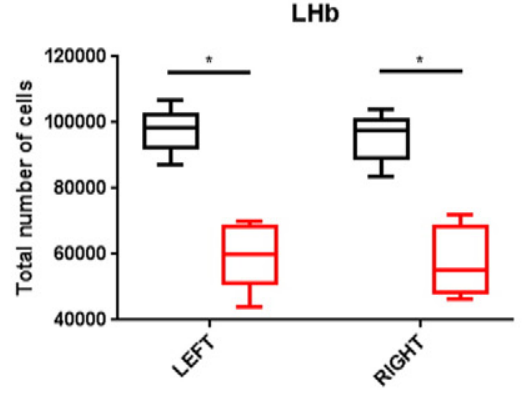

LHb

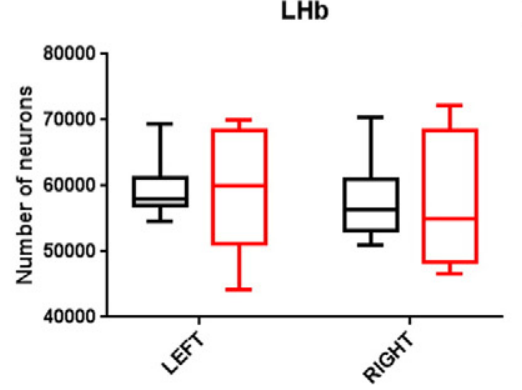

D

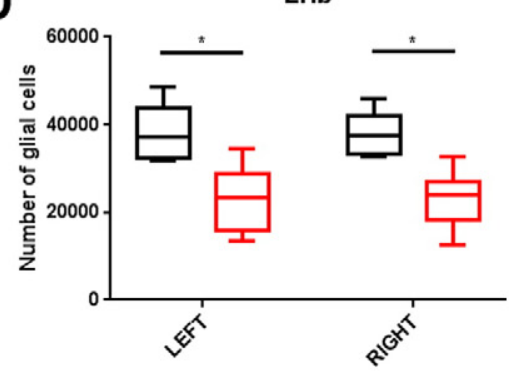

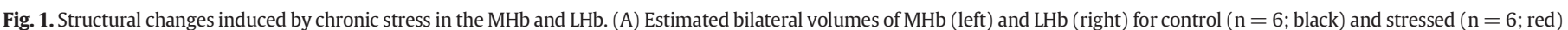

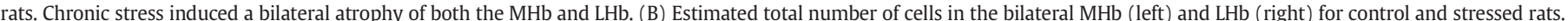

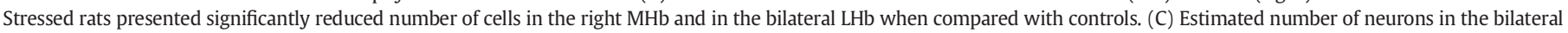

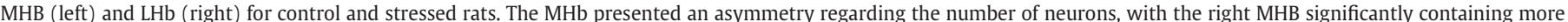

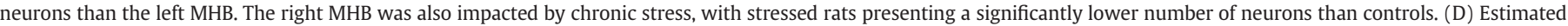

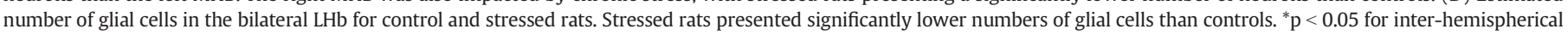
comparisons within the same group and inter-group comparisons. Error bars represent minimum and maximum values. 
changes on dendritic arborization, as in an additional set of control $(n=6)$ and stressed rats $(n=6)$, we did not observe significant differences in dendritic lengths of multipolar habenular neurons between experimental groups (Fig. 2).

As expected, stressed rats also presented concentrations of serum corticosterone significantly increased when compared with controls ( $p<0.01$; Fig. 3a) after the 28 days of exposure to stress. Interestingly, the levels of corticosterone presented high and significant negative correlations with the volumes of the bilateral MHb and LHb (Fig. 3c), the number of neurons in the right $\mathrm{MHb}$ and glial cells in the bilateral $\mathrm{LHb}$ (Supplementary Fig. 5). This means that rats that presented high levels of corticosterone at the end of the stress protocol were also the same that had lower volumes in the bilateral MHb and LHb, lower number of neurons in the right $\mathrm{MHb}$ and lower number of glial cells in the bilateral LHb.

Behaviorally, stressed rats displayed an anxiogenic phenotype, as they spent significantly less time in the open arms of the EPM than control rats ( $p<0.01$; Fig. 3b). No statistical differences were found between control and stress groups for the distance travelled in the EPM (control: $1511.63 \pm 74.87 \mathrm{~cm}$; stress $1620.83 \pm 72.77 \mathrm{~cm}$ ).

\subsection{Lesions of the habenula block stress-induced endocrine and behavioral responses}

In order to assess the effects of habenular lesion in stress-related anxiety behavior we performed bilateral electrolytic lesions in additional groups of rats. Part of these animals were subjected to the CUS protocol (lesion-stress, $\mathrm{n}=5$ ) while the remaining (lesion-control, $\mathrm{n}=4$ ) were handled during the same period. Blood for corticosterone assessment was drawn after 28 days of stress exposure. After blood collection animals were also exposed to the EPM and their performance compared with the previous control $(n=12)$ and stress $(n=12)$ groups.

Non-lesion stressed rats displayed, at the end of the stress protocol, serum corticosterone concentrations significantly higher than the ones displayed by non-lesion controls, lesion-controls and lesion-stress animals $(\mathrm{F}(3,33)=37.08, \mathrm{p}<0.0001$; post-hoc pairwise comparisons: control vs stress $\mathrm{p}<0.0001$; stress vs lesion-control $\mathrm{p}<0.0001$; stress vs lesion-stress $\mathrm{p}<0.0001$; Fig. 4a). There were no significant differences between the remaining groups.

Control, lesion-control and lesion-stress animals spent significantly more time in the open arms than non-lesioned stressed animals $(\mathrm{F}(3,33)=7.28, \mathrm{p}<0.001$; post-hoc pairwise comparisons: control vs stress $\mathrm{p}<0.01$; stress vs lesion-control $\mathrm{p}<0.01$; stress vs lesion-stress $\mathrm{p}<0.001$; Fig. $4 \mathrm{~b}$ ). Lesion-stress animals also spent significantly more time in the open arms than lesion-control animals ( $\mathrm{p}=0.02$ post-hoc pairwise comparison; Fig. 4b). No statistical differences were found between groups for the distance travelled in the EPM (control: $1511.63 \pm$ $74.87 \mathrm{~cm}$; stress $1620.83 \pm 72.77 \mathrm{~cm}$; lesion-control: $1722.78 \pm$ $63.94 \mathrm{~cm}$; lesion-stress: $1564.95 \pm 97.77 \mathrm{~cm}$ ).

\section{Discussion}

This study shows that chronic exposure to stress leads to an atrophy of the $\mathrm{LHb}$ and $\mathrm{MHb}$ bilaterally as well as to a reduction of the number of neurons in the right hemisphere $\mathrm{MHb}$ and the number of glial cells in the bilateral LHb. Specific structural chronic stress-induced changes involving volume, neuronal cell number and morphology variations have been shown to occur, for example, in the hippocampus-amygdala-prefrontal cortex (HPC-AMY-PFC) circuit, a key modulator of emotional behavior, among others (Cerqueira et al., 2007; Pêgo et al., 2008; Sousa et al., 2009; Pinto et al., 2015). Although it was known that the habenula, especially its lateral sub-region (LHb), reacts to stressful events (Wirtshafterm et al., 1994; Matsumoto and Hikosak, 2009) and that chronic stress can disrupt intracellular cascades in the LHb (Christensen et al., 2013), it had never been shown if repeated exposure to stress could impact on habenular structure/morphology as we show here. The current findings reveal that stress targets distinct cell populations in the habenula: while there is a decrease of neurons in the right $\mathrm{MHb}$, there is a decrease of glial cells in the bilateral LHb. Interestingly, we also found an hemispheric asymmetry in the MHb in this study: with the right $\mathrm{MHb}$ containing more neurons than its left counterpart in control rats. Although laterality of the habenula has been described in several species inducing differential expression of genes and connectivity (Hendricks and Jesuthasan, 2007; Dada et al. 2010) as well as responsiveness to stress (Facchin et al., 2015; Ichijo et al., 2015), this study shows for the first time, in a sample of 6 rats, that a structural difference also appears to exists in the rat.

\subsection{Chronic stress and cell loss in the habenula}

Chronic stress can target both neuronal and glial cell survival in various regions of the brain (Pittenger and Duman, 2008), as reported herein, despite the processes through which that occurs still being a matter of debate. Nevertheless, some hypothesis have been proposed, including: increased oxidative stress mediated by glucocorticoid-receptors (GR), highly expressed in both neurons and glia in various areas of the brain (Behl et al., 1996); impacts on neurogenesis (Duman, 2009); GR-induced glutamate deregulation and subsequent toxicity (Conrad, 2008); and loss of structural and trophic support (Lin and Koleske, 2010). In the context of the habenula, it is important to note that although GRs have not been found in this structure (Aronsson et al., 1988) and that the existence of MR or CRF receptors has never been reported, glucorticoid-induced receptor (GIR) - a receptor which has been proposed to also mediate stress-related glucocorticoid effects in the CNS -has been identified scattered throughout the habenula (Sah et al., 2005) and might be a possible mediator of the exposure to the increased corticosterone levels. Additionally, the habenula receives several projections, including glutamatergic ones, from limbic areas (Herkenham and Nauta, 1977) severely affected by glucorticoid-mediated stress which may have an impact on neuronal plasticity through
A

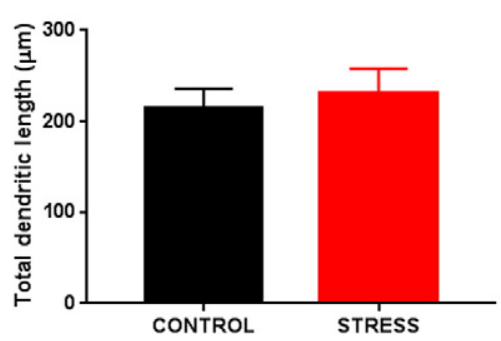

B
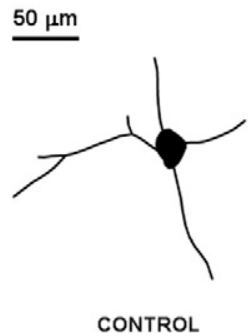

STRESS

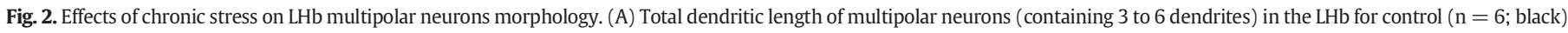

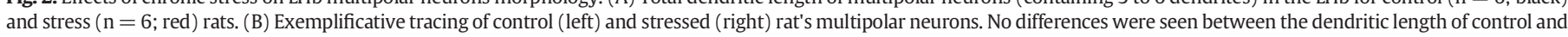
stressed rat's LHB multipolar neurons. Error bars represent sem. 
A
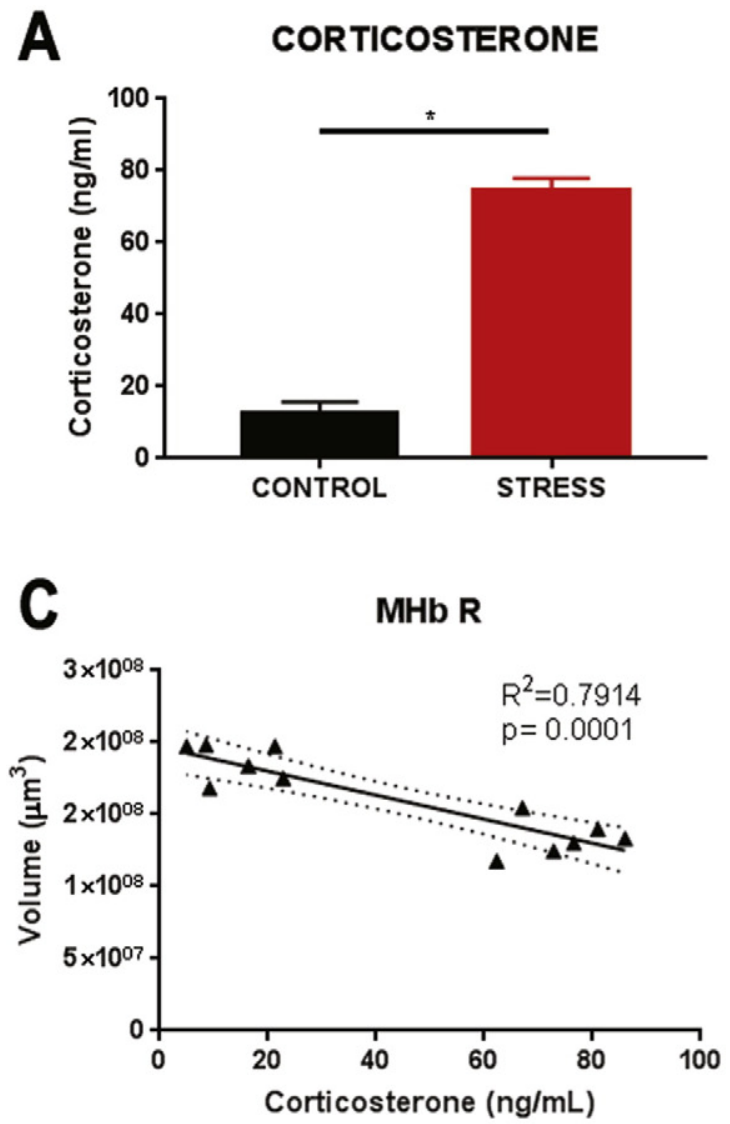

LHb R

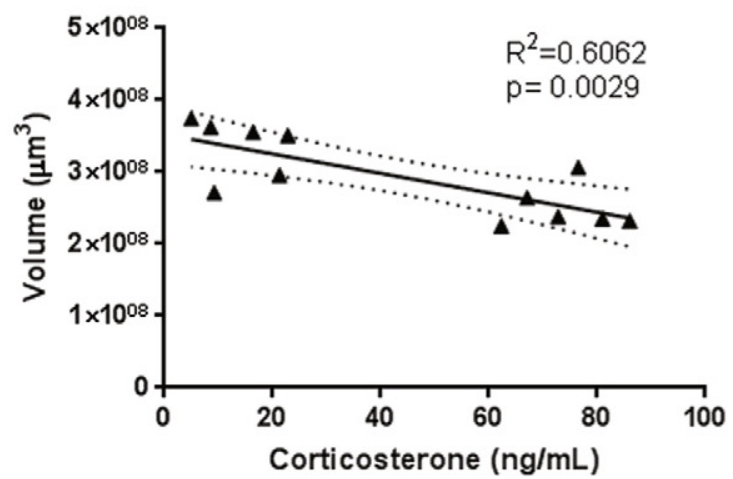

B

EPM [OPEN ARMS]

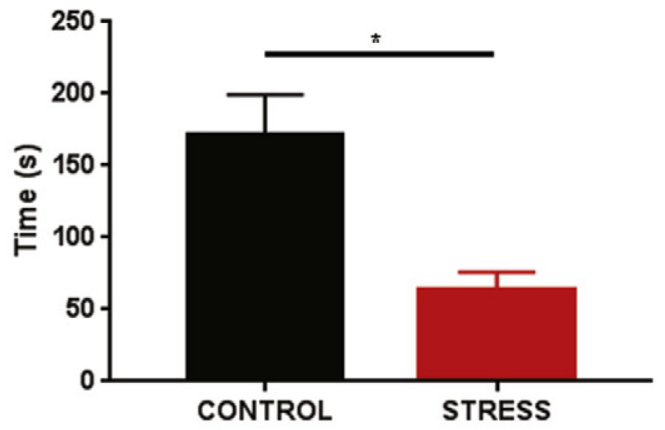

MHb L

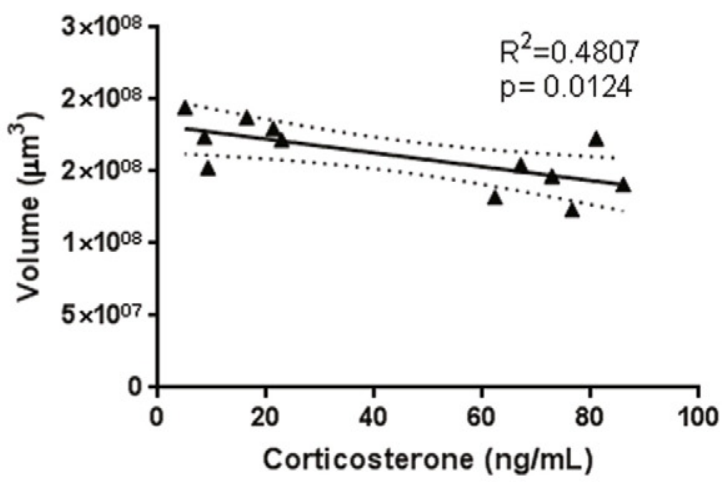

LHb L

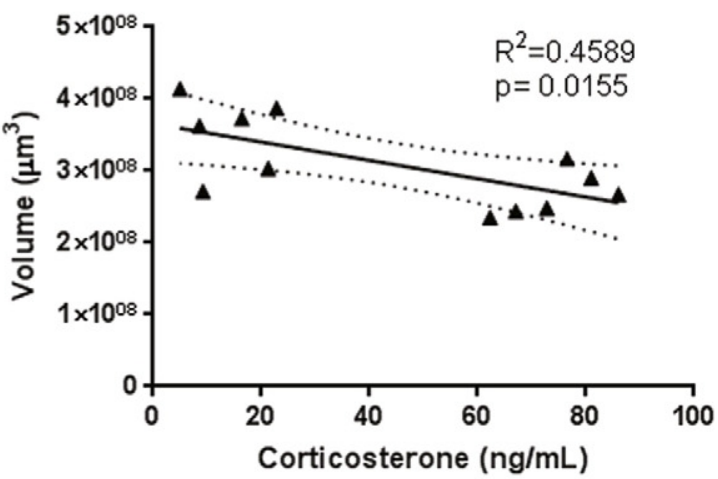

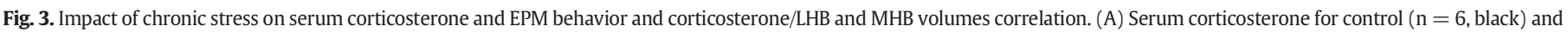

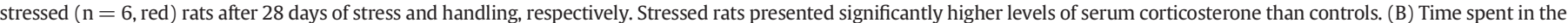

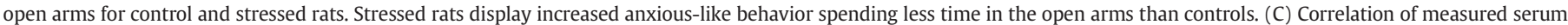

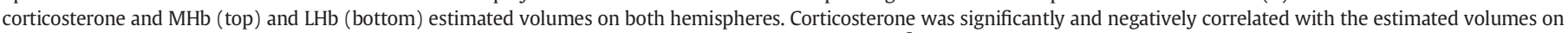
both hemispheres of the $\mathrm{MHb}$ and LHb. ${ }^{*} \mathrm{p}<0.05$ for inter-group comparisons. Error bars represent sem. $\mathrm{R}^{2}$ is goodness of fit and $\mathrm{p}$ is significance of linear regression.

disrupted neuromodulator-release (Sousa and Almeida, 2012). That the type of cells targeted by stress were different in the LHb and the MHb is surprising; however this may be a consequence of different expression of synaptic receptors in LHb and MHb (Meye et al., 2013) - although little is known regarding MHb synaptic profiling - and, above all, having segregated inputs from other brain areas which are probably affected differently throughout the stress exposure (Sousa, 2016). We also cannot entirely discard that stress may impact on glial cells on the MHb since the coefficient of error for a third of our estimates of MHb glial cells numbers were below the optimal range and these data was not taken into consideration.

\subsection{Habenula excitability and stress-related anxiety}

Stress-related changes in the structure of several brain areas have been shown to correlate with physiological and anxiety-like behavioral alterations across various contexts (Sousa and Almeida, 2012). The present study also shows that the stressed rats that displayed changes in volumes and cell numbers in both the LHb and $\mathrm{MHb}$ are the same that displayed increased corticosterone levels and increased response to anxiety-inducing stimuli. Chronic stress, in particular, is known to induce a state of hyper-reaction to anxiety-provoking contexts (Pêgo et al., 2008), such as the EPM. The modulation of behavioral inhibition in 
A

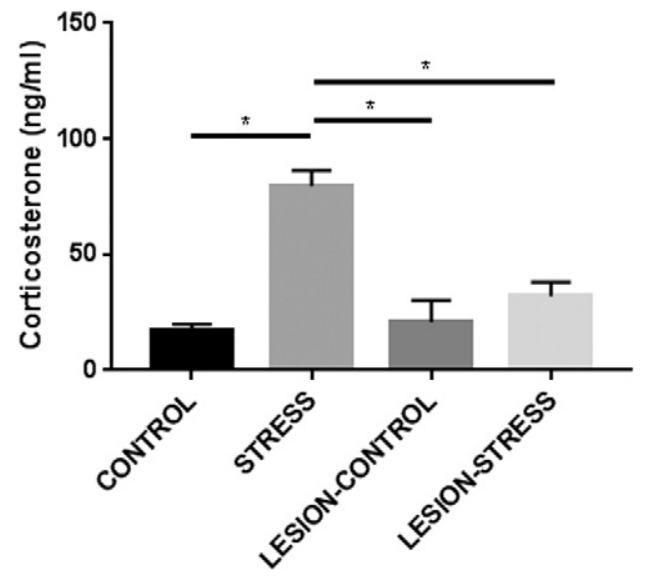

B

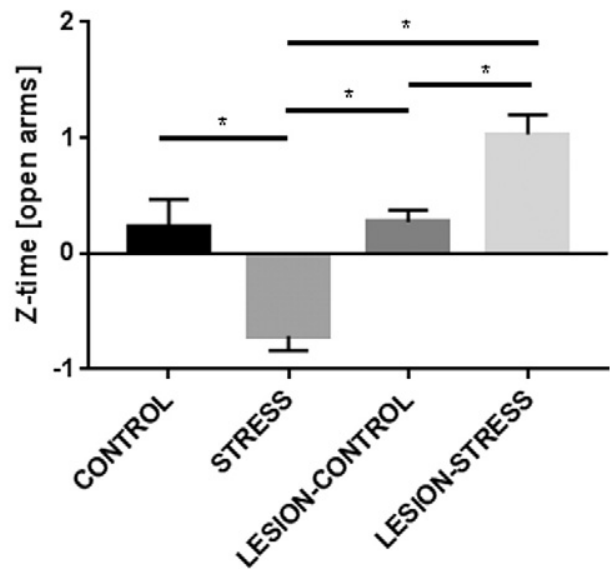

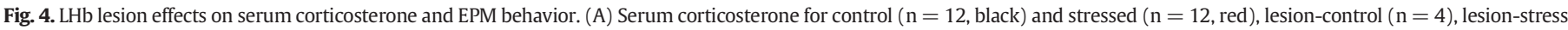

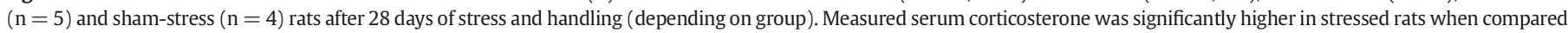

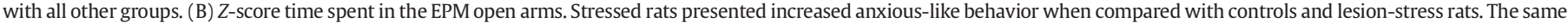
was observed for sham-stress animals when compared with lesion-stress animals. ${ }^{*} \mathrm{p}<0.05$ for post-hoc pairwise comparison. Error bars represent sem.

an aversive context or under acute stress through habenular function has been linked to serotonergic modulation (Teissier et al., 2015) and dopaminergic transmission (Hikosaka et al., 2008). Indeed, increased firing of LHb neurons, such as reported for a stressful event (Matsumoto and Hikosaka 2009), has been correlated with the reduction of neuronal firing of midbrain dopaminergic neurons (Matsumoto and Hikosaka, 2007; Stamatakis and Stuber, 2012) and with the inhibition of RN serotonergic neurons (Park, 1987; Stern et al., 1979) - although both the $\mathrm{LHb}$, directly and indirectly, and the MHb, indirectly, can act upon the RN with a net output that is not necessarily inhibitory (Proulx et al., 2014). Similarly, electric stimulation of the LHb induced a major suppression of dopaminergic neurons in VTA/SNc (Christoph et al., 1986; Ji and Shepard, 2007) and raphe units (Stern et al., 1979) - although previous reports have stated that high-frequency stimulation of the habenula produced anti-depressant effects in rats (Lim et al., 2015) and humans (Sartorius et al., 2010). Optogenetic activation of the LHb also induced active and passive behavioral avoidance (Stamatakis and Stuber, 2012). In contrast, lesions of the habenula can increase dopamine release in the cortex and striatum (Nishikawa et al., 1986; Lecourtier et al., 2008) and increased serotonin turnover in the dorsal RN (Yang et al., 2008) and are accompanied by exploratory disinhibition, even in an anxiogenic context (Murphy et al., 1996).

Coincidentally, monoaminergic dysfunction has been observed due to chronic stress exposure (Chaouloff et al., 1999; Pani et al., 2000) and in conditions of habenula hyperexcitability (Aizawa et al., 2013). Accordingly, an early hypothesis of habenula involvement in chronic stress alterations suggested that repetitive exposure to stressors would lead to hyperexcitability of the habenula with consequent dopaminergic and serotonergic activity reduction and increased behavioral inhibition (Hikosaka, 2010). There are several factors driving over-excitability in the habenula (Aizawa et al., 2013; Li et al., 2013) including glutamate accumulation in the synapse due to reduced clearing by glial transport (Cui et al., 2014). Thus, the glial loss that we observed in the LHb due to stress exposure may be contributing to the hyperexcitability of LHb neurons which, in turn, dysregulates the monoamines circuits and promotes the anxious behavior. Strikingly, depressive-like symptoms in a rodent model have been linked to glial dysfunction in the LHb affecting glutamate clearance and driving excitability of habenular neurons (Cui et al., 2014). The functional impact of the reduction of neurons in the right $\mathrm{MHb}$ is harder to clarify since the small size of this sub-region and its complexity have posed challenges to functional studies (Viswanath et al., 2014). Nevertheless, previous work showed that silencing the neural output of the dorsal MHb produced aversive and depressive behaviors (Hsu et al., 2014). Furthermore, the MHb projects almost uniquely to the interpeduncular nucleus (IPN), a region critically involved in regulating the RN (Herkenham and Nauta, 1979; Contestabile and Flumerfelt, 1981) and a recent study (Zhao-Shea et al., 2015) implicated MHb-IPN glutamatergic transmission in the modulation of anxiety.

Since the impact of chronic stress on habenular anatomy/morphology could just be a consequence of stress on the habenula and/or other regions projecting to it, and with no functional relevance, we decide to explore the involvement of the habenula in the stress-related anxiety response by lesioning the habenula in an additional group of rats that was submitted to the same CUS protocol as the group used for stereological and volume assessment. The lesions not only increased exploratory behavior in an anxiogenic context - in line with what was described above - but did it even after 4 weeks of unpredictable stress exposure. Thus, habenula lesion blocked the effects of chronic stress on EPM exploratory behavior. Due to the small group sizes of lesioned animals these results should be taken with caution, despite underlining the role of the habenula on exploratory activity and on the stress response.

Additionally, this study also shows that lesioning the habenula blocks the corticosterone rise typically observed after chronic exposure to stress (Sousa and Almeida, 2012). Chronic stress progressively impairs the inhibition of the hypothalamus-pituitary-adrenal (HPA) axis which leads to increasing levels of circulating corticosterone with further structural and functional consequences (Sousa and Almeida, 2012). The involvement of habenula activity in the modulation of HPA axis activity, measured indirectly by serum corticosterone levels, has never been clearly established. Conflicting results had previously shown that lesions of the habenula lowered the concentration of circulating corticosterone in rats as well as abolish the circadian fluctuations of this hormone (Montilla et al., 1982), on one hand; and that lesions of the fasciculus retroflexus, the de facto output of the LHb, would increase circulating corticosterone (Murphy et al., 1996), on the other hand. Several brain areas are known to be capable of activating and/or inhibiting the HPA axis directly and indirectly such as HPC-AMY-PFC circuit (Sousa and Almeida, 2012). Our study, despite showing that lesions of the habenula block the usual dysregulation of HPA axis activity brought about by chronic stress does not clarify how the habenula may be involved in this modulation. At this point we do not know if the habenula acts directly or indirectly in the HPA axis as additional causal studies would need to be performed - nevertheless, both possibilities are open since the LHb projects to several areas of the hypothalamus 
(Herkenham and Nauta, 1979) and can exert monoamine dependent modulation in several brain areas capable of regulating the axis.

\section{Conclusions}

The main findings herein reported may appear contradictory: on one hand, atrophy and cell number reduction on both the $\mathrm{MHb}$ and $\mathrm{LHb}$ are a consequence of chronic exposure to stress and correlate with anxious behavior; and that, on another hand, fully ablating the LHb blocks the stress effects both physiologically and behaviorally. However, what these results suggest is that the action of chronic stress on the habenula is complex - targeting, for example, different cell populations on each sub-region through mechanisms that have not yet been unveiled, as discussed above - and that the normal and pathological behavioral outcomes most likely depend on the combined net output of these two subregions. Taken together, these results reinforce the need for future studies in order to dissect the important role of the habenula in the stress and anxiety responses. Given that neuroanatomic differences can underlie stress susceptibility and stress resilience and that the habenula has been receiving increased attention as a promising therapeutic target for depression and anxiety this study further highlights the clinical relevance of the habenula in normal and pathological conditions.

All applicable international, national, and/or institutional guidelines for the care and use of animals were followed.

Supplementary data to this article can be found online at http://dx. doi.org/10.1016/j.expneurol.2016.12.003.

\section{Acknowledgements}

The authors would like to acknowledge Prof. Sharif Taha formerly of University of Utah for providing training and technical expertise on habenula research; Ana Lima and Mónica Dias for histological processing of samples; and Diana Afonso and Ana Veloso for help with the stress protocol. LRJ was supported by fellowships: UMINHO/BPD/27/ 2013 funded by CCDR-N and Programa Operacional Região Norte (ON.2) from QREN/FEDER; 2014/CON3/CAN23 from Fundação LusoAmericana; and UMINHO/BPD/63/2015 from Fundação Calouste Gulbenkian funded project (contract grant number P-139977). AN was supported by a fellowship from project ANR/NEU-OSD/0258/2012 funded by Fundação para a Ciência e Tecnologia (FCT) and Agence Nationale de la Recherche (ANR). Financial support for this work was provided by FEDER funds through the Operational Programme Competitiveness Factors - COMPETE and National Funds through FCT - Foundation for Science and Technology under the project POCI-01-0145FEDER-007038; and by the project NORTE-01-0145-FEDER-000013, supported by Norte Portugal Regional Operational Programme (NORTE 2020), under the PORTUGAL 2020 Partnership Agreement, through the European Regional Development Fund (ERDF).

\section{References}

Acer, N., Sahin, B., Usanmaz, M., Tatoğlu, H., Irmak, Z., 2008. Comparison of point counting and planimetry methods for the assessment of cerebellar volume in human using magnetic resonance imaging: a stereological study. Surg. Radiol. Anat. 30 (4), 335-339.

Aizawa, H., Cui, W., Tanaka, K., Okamoto, H., 2013. Hyperactivation of the habenula as a link between depression and sleep disturbance. Front. Hum. Neurosci. 7. http://dx. doi.org/10.3389/fnhum.2013.00826.

Amo, R., et al., 2014. The habenulo-raphe serotonergic circuit encodes an aversive expec tation value essential for adaptive active avoidance of danger. Neuron 84 (5), 1034-1048.

Andres, K.H., von Düring, M., Veh, R.W., 1999. Subnuclear organization of the rat habenular complexes. J. Comp. Neurol. 407 (1), 130-150.

Aronsson, M., et al., 1988. Localization of glucocorticoid receptor mRNA in the male rat brain by in situ hybridization. Proc. Natl. Acad. Sci. U. S. A. 85 (23), 9331-9335.

Bambico, F.R., Nguyen, N.T., Gobbi, G., 2009. Decline in serotonergic firing activity and desensitization of 5-HT1A autoreceptors after chronic unpredictable stress. Eur. Neuropsychopharmacol. 19 (3), 215-228.

Behl, C., et al., 1996. Glucocorticoids enhance oxidative stress-induced cell death in hippocampal neurons in vitro. Behav. Brain Res. 81 (1-2), 43-52.
Cerqueira, J.J., Mailliet, F., Almeida, I.F., Jay, T.M., Sousa, N., 2007. The prefrontal cortex as a key target of the maladaptive response to stress. J. Neurosci. 27 (11), 2781-2787.

Chaouloff, F., Berton, O., Mormède, P., 1999. Serotonin and stress. Neuropsychopharmacology 21 (Suppl. 2), 28S-32S.

Christensen, T., Jensen, L., Bouzinova, E.V., Wiborg, O., 2013. Molecular profiling of the lateral habenula in a rat model of depression. PLoS ONE 8 (12). http://dx.doi.org/10. 1371/journal.pone.0080666.

Christoph, G.R., Leonzio, R.J., Wilcox, K.S., 1986. Stimulation of the lateral habenula inhibits dopamine-containing neurons in the substantia nigra and ventral tegmental area of the rat. J. Neurosci. 6 (3), 613-619.

Conrad, C.D., 2008. Chronic stress-induced hippocampal vulnerability: the glucocorticoid vulnerability hypothesis. Rev. Neurosci. 19 (6), 395-411.

Contestabile, A., Flumerfelt, B.A., 1981. Afferent connections of the interpeduncular nucleus and the topographic organization of the habenulo-interpeduncular pathway: an HRP study in the rat. J. Comp. Neurol. 196 (2), 253-270.

Cotter, D., et al., 1999. The assessment of postmortem brain volume; a comparison of stereological and planimetric methodologies. Neuroradiology 41 (7), 493-496.

Cui, W., et al., 2014. Glial dysfunction in the mouse habenula causes depressive-like behaviors and sleep disturbance. J. Neurosci. 34 (49), 1685-16273.

Dadda, M., Domenichini, A., Piffer, L., Argenton, F., Bisazza, A., 2010. Early differences in epithalamic left-right asymmetry influence lateralization and personality of adult zebrafish. Behav. Brain Res. 206 (2), 208-215.

Davanlou, M., Smith, D.F., 2004. Unbiased stereological estimaton of different cell types in rat cerebral cortex. Image Anal. Stereol. 23, 1-11.

Duman, R.S., 2009. Neuronal damage and protection in the pathophysiology and treatment of psychiatric illness: stress and depression. Dialogues Clin. Neurosci. 11 (3), 239-255.

Facchin, L., Duboué, E.R., Halpern, M.E., 2015. Disruption of epithalamic left-right asymmetry increases anxiety in Zebrafish. J. Neurosci. 35 (48), 15847-15859.

Fakhoury, M., López, S.D., 2014. The role of habenula in motivation and reward. Adv. Neurosci. http://dx.doi.org/10.1155/2014/862048.

Ferraro, G., Montalbano, M.E., Sardo, P., La Grutta, V., 1996. Lateral habenular influence on dorsal raphe neurons. Brain Res. Bull. 41 (1), 47-52.

García-Amado, M., Prensa, L., 2012. Stereological analysis of neuron, glial and endothelial cell numbers in the human amygdaloid complex. PLoS ONE 7 (6):2012. http://dx.doi. org/10.1371/journal.pone.0038692.

Gibb, R., Kolb, B., 1986. A method for vibratome sectioning of Golgi-Cox stained whole rat brain. J. Neurosci. Methods 79 (1), 1-4.

Haack, A.K., et al., 2014. Lesions of the lateral habenula increase voluntary ethanol consumption and operant self-administration, block yohimbine-induced reinstatement of ethanol seeking, and attenuate ethanol-induced conditioned taste aversion. PLoS ONE 9 (4). http://dx.doi.org/10.1371/journal.pone.0092701.

Healy-Stoffel, M., Omar Ahmad, S., Stanford, J.A., Levant, B., 2001. Differential effects of intrastriatal 6-hydroxydopamine on cell number and morphology in midbrain dopaminergic subregions of the rat. Brain Res. 1574, 113-119.

Hendricks, M., Jesuthasan, S., 2007. Asymmetric innervation of the habenula in zebrafish. J. Comp. Neurol. 502 (4), 611-619.

Herkenham, M., Nauta, W.J., 1977. Afferent connections of the habenular nuclei in the rat. A horseradish peroxidase study, with a note on the fiber-of-passage problem. J. Comp. Neurol. 173 (1), 123-146.

Herkenham, M., Nauta, W.J., 1979. Efferent connections of the habenular nuclei in the rat. J. Comp. Neurol. 187 (1), 19-47.

Hikosaka, O., 2010. The habenula: from stress evasion to value-based decision-making. Nat. Rev. Neurosci. 11 (7), 503-513.

Hikosaka, O., Sesack, S.R., Lecourtier, L., Shepard, P.D., 2008. Habenula - crossroad between the basal ganglia and the limbic system. J. Neurosci. 28 (46), 11825-11829.

HJG, G., EBV, J., Kiêu, K., Nielsen, J., 1999. The efficacy of systemic sampling in stereology reconsidered. J. Microsc. 193 (3), 199-211.

Hsu, Y.W., et al., 2014. Role of the dorsal medial habenula in the regulation of voluntary activity, motor function, hedonic state, and primary reinforcement. J. Neurosci. 34 (34), 11366-11384.

Ichijo, H., et al., 2015. Lateralization, maturation, and anteroposterior topography in the lateral habenula revealed by ZIF268/EGR1 immunoreactivity and labeling history of neuronal activity. Neurosci. Res. 95, 27-37.

Jacinto, L.R., et al., 2013. Stress affects theta activity in limbic networks and impairs novelty-induced exploration and familiarization. Front. Behav. Neurosci.:7 http://dx.doi. org/10.3389/fnbeh.2013.00127.

Ji, H., Shepard, P.D., 2007. Lateral habenula stimulation inhibits rat midbrain dopamine neurons through a GABA(A) receptor-mediated mechanism. J. Neurosci. 27 (26), 6923-6930.

Kim, U., Chang, S.Y., 2005. Dendritic morphology, local circuitry, and intrinsic electrophysiology of neurons in the rat medial and lateral habenular nuclei of the epithalamus. J. Comp. Neurol. 483 (2), 236-250.

Lecourtier, L., Defrancesco, A., Moghaddam, B., 2008. Differential tonic influence of lateral habenula on prefrontal cortex and nucleus accumbens dopamine release. Eur. J. Neurosci. 27 (7), 1755-1762.

Lee, E.H., Huang, S.L., 1988. Role of lateral habenula in the regulation of exploratory behavior and its relationship to stress in rats. Behav. Brain Res. 30 (3), 265-271.

$\mathrm{Li}$, K., et al., 2013. $\beta$ CaMKII in lateral habenula mediates core symptoms of depression. Science 341, 1016-1020

Lim, L.W., et al., 2015. Electrical stimulation alleviates depressive-like behaviors of rats: investigaton of brain targets and potential mechanisms. Transl. Psychiatry 5 (3). http://dx.doi.org/10.138/tp.2015.24

Lin, Y.C., Koleske, A.J., 2010. Mechanisms of synapse and dendrite maintenance and their disruption in psychiatric and neurodegenerative disorders. Annu. Rev. Neurosci. 33, 349-378. 
Matsumoto, M., Hikosak, O., 2009. Representation of negative motivational value in the primate lateral habenula. Nat. Neurosci. 12 (1), 77-84.

Matsumoto, M., Hikosaka, O., 2007. Lateral habenula as a source of negative reward signals in dopamine neurons. Nature 447 (7148), 1111-1115.

Meye, F.J., Lecca, S., Valentinova, K., Mameli, M., 2013. Synaptic and cellular profile of neurons in the lateral habenula. Front. Hum. Neurosci. 7. http://dx.doi.org/10.3389/ fnhum.2013.00860.

Montilla, P., Muñoz, M.C., Bellido, M.C., 1982. Influence of the habenular complex on the cyclic secretion of plasma corticosterone in rats. Rev. Esp. Fisiol. 38 (3), 333-338.

Murphy, C.A., AM, D.C., Haun, F., Murray, M., 1996. Lesion of the habenular efferent pathway produces anxiety and locomotor hyperactivity in rats: a comparison of the effects of neonatal and adult lesions. Behav. Brain Res. 81 (1-2), 43-52.

Nishikawa, T., Fage, D., Scatton, B., 1986. Evidence for, and nature of, the tonic inhibitory influence of habenulointerpeduncular pathways upon cerebral dopaminergic transmission in the rat. Brain Res. 373 (1-2), 324-336.

Ootsuka, Y., Mohammed, M., 2015. Activation of the habenula complex evokes autonomic physiological responses similar to those associated with emotional stress. Phys. Rep. 3 (2). http://dx.doi.org/10.14814/phy2.12297.

Pani, L., Porcella, A., Gessa, G.L., 2000. The role of stress in the pathophysiology of the dopaminergic system. Mol. Psychiatry 5 (1), 14-21.

Park, M.R., 1987. Monosynaptic inhibitory postsynaptic potentials from lateral habenula recorded in dorsal raphe neurons. Brain Res. Bull. 19 (5), 581-586.

Paxinos, G., Watson, C., 2006. The Rat Brain in Stereotaxic Coordinates. sixth ed. Academic Press (Elsevier), London.

Pêgo, J.M., et al., 2008. Dissociation of the morphological correlates of stress-induced anxiety and fear. Eur. J. Neurosci. 27 (6), 1503-1516.

Pelvig, D.P., Pakkenberg, H., Pakkenberg, B., 2008. Neocortical glial cell numbers in human brains. Neurobiol. Aging 29 (11), 1754-1762.

Pinto, V., et al., 2015. Differential impact of chronic stress along the hippocampal dorsalventral axis. Brain Struct. Funct. 220 (2), 1205-1512.

Pittenger, C., Duman, R.S., 2008. Stress, depression, and neuroplasticity: a convergence of mechanisms. Neuropsychopharmacology 33 (1), 88-109.

Proulx, C.D., Hikosaka, O., Malinow, R., 2014. Reward processing by the lateral habenula in normal and depressive behaviors. Nat. Neurosci. 17 (9), 1146-1152.

Russo, S.J., Nestler, E.J., 2013. The brain reward circuitry in mood disorders. Nat. Rev. Neurosci. 14 (9), 609-625.

Sachs, B.D., Ni, J.R., Caron, M.G., 2015. Brain 5-HT deficiency increases stress vulnerability and impairs antidepressant responses following psychosocial stress. Proc. Natl. Acad. Sci. U. S. A. 112 (8), 2557-2562.

Sah, R., et al., 2005. Expression of the glucocorticoid-induced receptor mRNA in rat brain. Neuroscience 133 (1), 281-292.

Sala, C., Segal, M., 2014. Dendritic spines: the locus of structural and functional plasticity. Physiol. Rev. 94 (1), 141-188.

Sartorius, A., et al., 2010. Remission of major depression under deep brain stimulation of the lateral habenula in a therapy-refractory patient. Biol. Psychiatry 67 (2), e9-e11.

Sousa, N., 2016. The dynamics of the stress neuromatrix. Mol. Psychiatry 21 (3), 302-312.

Sousa, N., Almeida, O.F., 2012. Disconnection and reconnection: the morphological basis of (mal)adaptation to stress. Trends Neurosci. 35 (12), 742-751.
Sousa, N., Almeida, O.F., Wotjak, C.T., 2006. A hitchhiker's guide to behavioral analysis in laboratory rodents. Genes Brain Behav. 5 (Suppl. 2), 5-24.

Sousa, N., Lukoyanov, N.V., Madeira, M.D., Almeida, O.F., Paula-Barbosa, M.M., 2009. Reorganization of the morphology of hippocampal neurites and synapses after stress-induced damage correlates with behavioral improvement. Neuroscience 97 (2), 253-266.

Stamatakis, A.M., Stuber, G.D., 2012. Activation of lateral habenula inputs to the ventra midbrain promotes behavioral avoidance. Nat. Neurosci. 15 (8), 1105-1107.

Stephenson-Jones, M., Floros, O., Robertson, B., Grillner, S., 2012. Evolutionary conservation of the habenular nuclei and their circuitry controlling the dopamine and $5-$ hydroxytryptophan (5-HT) systems. Proc. Natl. Acad. Sci. U. S. A. 109 (3), E164-E173 (2012).

Stern, W.C., Johnson, A., Bronzino, J.D., Morgane, P.J., 1979. Effects of electrical stimulation of the lateral habenula on single-unit activity of raphe neurons. Exp. Neurol. 65 (2) 326-342.

Teissier, A., et al., 2015. Activity of Raphé serotonergic neurons controls emotional behaviors. Cell Rep. 13 (9), 1965-1976.

Tian, J., Uchida, N., 2015. Habenula lesions reveal that multiple mechanisms underlie dopamine prediction errors. Neuron 87 (6), 1304-1316.

Uylings, H.B., Ruiz-Marcos, A., van Pelt, J., 1986. The metric analysis of three-dimensional dendritic tree patterns: a methodological review. J. Neurosci. Methods 8 (1-2) $127-151$.

Vertes, R.P., 1991. A PHA-L analysis of ascending projections of the dorsal raphe nucleus in the rat. J. Comp. Neurol. 313 (4), 643-668.

Viswanath, H., Carter, A.Q., Baldwin, P.R., Molfese, D.L., Salas, R., 2014. The medial habenula: still neglected. Front. Hum. Neurosci. 7. http://dx.doi.org/10.3389/fnhum. 2013.00931 (eCollection 2013., 2014).

West, M.J., Slomianka, L., Gundersen, H.J., 1991. Unbiased stereological estimation of the total number of neurons in the subdivisions of the rat hippocampus using the optical fractionator. Anat. Rec. 231 (4), 482-497.

Winter, C., Vollmayr, B., Djodari-Irani, A., Klein, J., Sartorius, A., 2011. Pharmacological inhibition of the lateral habenula improves depressive-like behavior in an animal model of treatment resistant depression. Behav. Brain Res. 216 (1), 463-465.

Wirtshafterm, D., Asin, K.E., Pitzer, M.R., 1994. Dopamine agonists and stress produce different patterns of Fos-like immunoreactivity in the lateral habenula. Brain Res. 633 (1-2), 21-26

Yang, L.M., Hu, B., Xia, Y.H., Zhang, B.L., Zhao, H., 2008. Lateral habenula lesions improve the behavioral response in depressed rats via increasing the serotonin level in dorsal raphe nucleus. Behav. Brain Res. 188 (1), 84-90.

Zhang, R., Oorschot, D.E., 2006. Total number of neurons in the habenular nuclei of the rat epithalamus: a stereological study. J. Anat. 208 (5), 577-585.

Zhao, H., Zhang, B.L., Yang, S.J., Rusak, B., 2015. The role of lateral habenula-dorsal raphe nucleus circuits in higher brain functions and psychiatric illness. Behav. Brain Res. 277, 89-98.

Zhao-Shea, R., et al., 2015. Increased CRF signalling in a ventral tegmental areainterpeduncular nucleus-medial habenula circuit induces anxiety during nicotine withdrawal. Nat. Commun. 6. http://dx.doi.org/10.1038/ncomms7770. 